\title{
A AVALIAÇÃO HEURÍSTICA APLICADA AO PLANO DE SUPERFÍCIE NO MÉTODO DE PROJETO CENTRADO NO USUÁRIO, DE JESSE JAMES GARRETT
}

\section{HEURISTIC EVALUATION APPLIED TO SURFACE PLAN, ON USER-CENTERED DESIGN METHOD, BY J. J. GARRETT}

\author{
Airton Jordani Jardim Filho \\ Universidade do Estado de Santa Catarina \\ airtonjordani@gmail.com \\ Sandra Regina Ramalho e Oliveira \\ Universidade do Estado de Santa Catarina \\ ramalho@floripa.com.br \\ Flavio Anthero Nunes Vianna dos Santos \\ Universidade do Estado de Santa Catarina \\ flavioanvs@hotmail.com
}

\begin{abstract}
Resumo: O objetivo deste artigo é propor a aplicação da técnica de avaliação heurística de interface já na etapa de Superfície, antes mesmo do projeto ser disponibilizado ao usuário final, no método de projeto de interfaces web centrado no usuário de Jesse James Garrett, em função da provável redução do retrabalho e, por consequência, do custo de desenvolvimento de projeto, de uma maneira geral.
\end{abstract}

Palavras-chave: Avaliação, heurística, interface, método e projeto.

Abstract: This paper aims to propose the application of heuristic evaluation technique on the Surface Plan, of Jesse James Garrett's user-centered design method for web based interfaces, likely due to the reduction of rework and the cost of project development as well.

Key-words: Evalutation, heuristic, interface, method, project. 


\section{INTRODUÇÃO}

Cada vez mais, a usabilidade torna-se um requisito básico para o desenvolvimento de projetos de interfaces digitais. Segundo LYNCH \& HORTON (2008, p.52),

o método mais comum para atingir a usabilidade é o chamado design centrado no usuário (UCD). UCD inclui métodos orientados ao usuário, tais como análise de tarefas, grupos focais, e testes de usuário para entender suas necessidades e refinar projetos baseando-se no retorno que o usuário fornece.

A partir de uma análise do método User-Centered Design for the Web, de J. J. Garrett (2003), para o desenvolvimento de projetos dígito-virtuais, percebemos que este método, apesar de bastante aceito e utilizado, pode receber contribuições com o intuito de ampliá-lo para seu uso tanto no ensino do projeto de interfaces, quanto no seu uso prático do dia-a-dia.

Prova disso foi a proposta de inserção de uma sexta etapa, intitulada Execução, por Heli Meurer e Daniela Szabluk, em artigo publicado nos Anais do 9. ERGODESIGN | 9. USIHC (2009) e na Revista Ação Ergonômica, da ABERGO (2010).

O Projeto E, segundo seus autores (2010, p. 2) objetiva "guiar e otimizar o desenvolvimento de projetos de interfaces gráficas amigáveis para diferentes sistemas e produtos interativos dígito-virtuais e tem sua estrutura baseada no método de Garrett".

No entanto, a avaliação da interface gerada pelo quinto plano de GARRETT (2003) não é abordada, detalhadamente, por nenhum dos autores. MEURER \& SZABLUK (2009), inclusive, recomendam que o momento em que o produto deve passar por uma avaliação heurística e testes com usuários se dá posteriormente à sua fase de acabamento, com todas as suas ferramentas e funcionalidades ativas, para identificar e corrigir possíveis erros programacionais e de usabilidade.

\section{O MÉTODO DE PROJETO DE GARRETT E MEURER \& SZABLUK}

Garrett propôs o método no livro "The Elements of User Experience: Usercentered design for the web" (2003). Sua popularidade se deve, provavelmente, à sua simplicidade, fácil adaptação e, sobretudo, pioneirismo. O autor propõe cinco planos (ou etapas) para o processo de produção de um website: estratégia, escopo, estrutura, esqueleto e superfície.
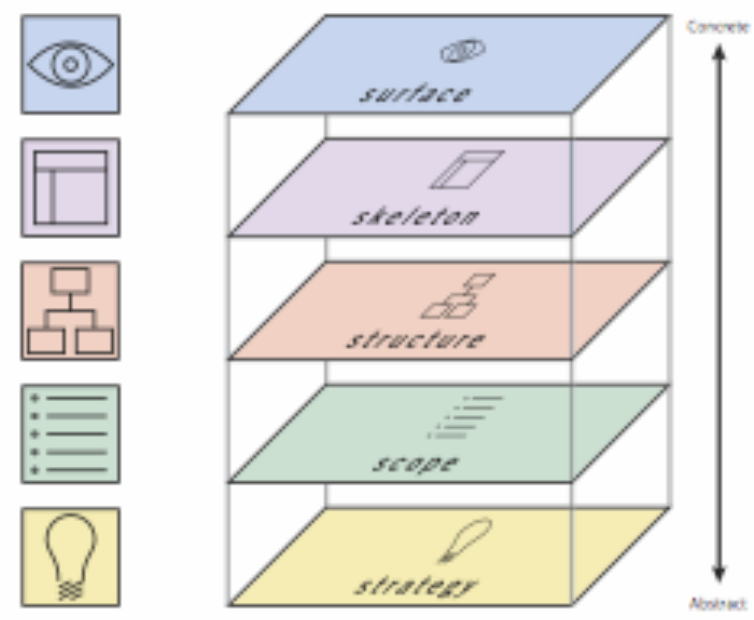
Figura 1 - Os cinco planos da metodologia de J.J. Garrett (2003)

A ideia é construir o website usando uma abordagem de baixo para cima (bottom-up), começando com conceitos abstratos e chegando a definições concretas, cada vez mais ricas em detalhes. O próprio diagrama se assemelha a um prédio, cujas etapas seriam os andares. A etapa de estratégia, por exemplo, seria a fundação do prédio e uma alteração nela teria efeitos drásticos nos demais andares. A mudança no escopo afeta a estrutura e assim por diante.

Isso não significa que cada decisão sobre um plano tem que ser necessariamente feita antes que se comece a definir o plano subsequente. Em certos momentos, é necessário reavaliar decisões anteriores e mudar o rumo do projeto. Por isso, é importante que o projeto entre em um ciclo constante de avaliação para que se identifiquem desvios antes que seja tarde demais para mudar.

\subsection{Estratégia}

Desta forma, o primeiro passo para o desenvolvimento do projeto é justamente traçar a estratégia a ser adotada. Define-se o quê e para quem se quer comunicar. Envolve entender as necessidades do usuário (influência externa) e os objetivos do site (influência interna). Segundo GARRETT (2011, p. 21),

\footnotetext{
esta estratégia incorpora não apenas o que as pessoas que dirigem o site querem dele, mas o que os usuários querem, na verdade. As necessidades dos usuários são conhecidas a partir da definição dos objetivos do site através da análise das características do públicoalvo. Já os objetivos do site são conhecidos através da definição do que se quer comunicar através do site e qual o propósito do seu desenvolvimento.
}

\subsection{Escopo}

O passo seguinte é a definição das características do produto. Aqui se estabelecem quais serão, efetivamente, os conteúdos do site. De que forma serão supridas as necessidades do usuário. Cabe ressaltar que tão ou mais importante do que deixar claro aquilo que faz parte do projeto, é definir o que não faz.

Quando definimos o escopo de nosso projeto definimos o foco de nosso trabalho, deixando absolutamente claro aquilo que será incluído e aquilo que será excluído, por sua vez. Um escopo malfeito pode acarretar retrabalho ou prejuízo para quem está desenvolvendo o projeto, uma vez que quando não existem fronteiras bem definidas, damos margem à especulação e à dúvida.

\subsection{Estrutura}

Segundo a proposta de metodologia de trabalho de Garrett, planejar a estrutura nada mais é do que organizar a hierarquia de páginas, os caminhos entre elas. Normalmente descrita através de um organograma ou de um fluxograma, a estrutura da uma visão geral do website.

Aqui entra o trabalho da Arquitetura da Informação, atuando no planejamento estrutural do espaço reservado à informação no site, de forma que se crie uma maneira intuitiva de acesso ao conteúdo. Arquitetura de informação, para BRINK, GERGLE e WOOD (2002) apud SANTA ROSA \& MORAES (2008, p. 24), "se refere à 
estrutura da organização de um website, especialmente como as diferentes páginas do site se relacionam entre si. Ela envolve aspectos tais como análise e planejamento, organização das páginas, rotulagem, técnicas de busca, projeto da navegação e dá dicas para ajudar os usuários a se orientarem."

\subsection{Esqueleto}

O esqueleto nada mais é do que o desenho estrutural das telas, dos menus e formulários. Essa etapa da metodologia se preocupa em erguer o esqueleto da página, sua estrutura "óssea". Por trás da superfície, que é a próxima e última etapa da metodologia, está o esqueleto que dá a sustentação à página.

No esqueleto, surge uma bifurcação conceitual: o design de navegação e o de informação. $O$ design de navegação trata da disposição dos elementos da interface de forma a facilitar a navegação do usuário através da arquitetura da informação. Já o design da Informação define como apresentar a informação de forma a facilitar o entendimento por parte do usuário. 0 método de desenho do esqueleto, para que este possa ser representado graficamente chama-se wireframe.

\subsection{Superfície}

A quinta e última etapa da metodologia é chamada de superfície. Aqui entra, efetivamente, o trabalho do design visual.

No topo do modelo dos cinco planos, foca-se nos aspectos do site que nosso usuário vai perceber de imediato: o design visual. Aqui, conteúdo, funcionalidade e estética unem-se para produzir um design bem-acabado que supre os Objetivos de todos os outros quatro planos. (GARRETT, 2003, p. 133)

A superfície nada mais é do que o fechamento, a partir de um trabalho em design visual, dos elementos que compõem o site como um todo. A comunicação visual exerce, então, um papel importante no planejamento de qualquer website, pois é responsável pela primeira impressão que o usuário tem de todo o trabalho de projeto e planejamento, uma espécie de porta de entrada.

\subsection{O Projeto E, de Meurer \& Szabluk}

Segundo BOMFIM (1995, p. XX), os métodos em design apresentam três grandes fases: a fase analítica, a fase criativa e a fase executiva. Na fase analítica é definido o problema. Os requisitos do projeto são traçados e é onde surge o conceito do projeto. A fase seguinte, que o autor chama de criativa, é onde as alternativas são geradas, sendo selecionadas as melhores e depuradas até que se chegue àquela mais adequada à solução. Por fim, a terceira seria a fase executiva, onde se faz o detalhamento do projeto para a execução e o acompanhamento da produção. Assim sendo, o método de Garrett poderia incluir em suas etapas um último plano destinado à execução, uma vez que o método original restringe-se apenas ao projeto e não à execução. Nesse sentido, o Projeto E, de MEURER \& SZABLUK (2009) faz importantes contribuições ao método. Definida pelos seus autores como uma "metodologia constituída de conceitos, definições, métodos e processos de autores consagrados em design, estruturados de acordo com as etapas sugeridas por Garrett", sua proposta acrescenta uma sexta etapa ao método, chamada de etapa de execução. 


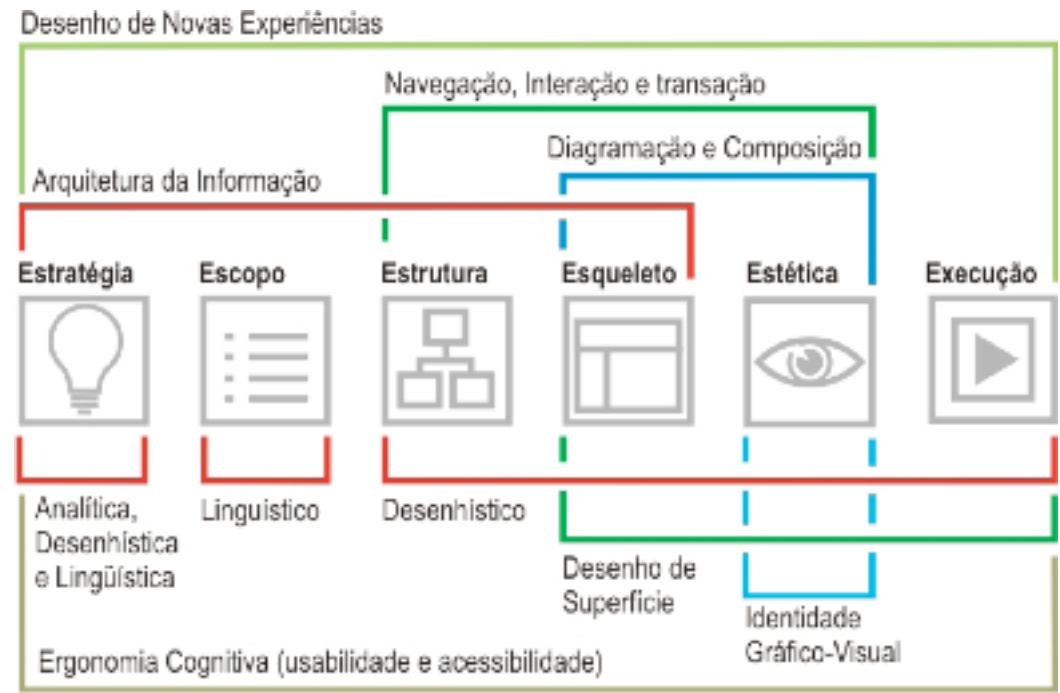

Figura 2 - Diagrama do Projeto E, contemplando uma sexta etapa na proposta inicial de GARRETT (2003), por MEURER \& SZABLUK (2009)

A aplicação do Projeto E contribui para a potencialização dos recursos da world wide web ou de interfaces diversas. Além disso, o método incentiva o profissional a preocupar-se com aspectos ergonômicos, de usabilidade e de acessibilidade, imprescindíveis aos projetos dígito-virtuais e muitas vezes considerados menos importantes."

\section{A AVALIAÇÃO HEURÍSTICA}

Uma importante técnica para avaliação de interfaces é a chamada avaliação heurística. Segundo SALMON (2004), uma grande vantagem do uso da análise heurística consiste na simplicidade e no fato de que pode ser conduzida, também, durante o processo projetivo, em suas diversas etapas.

A avaliação Heurística surge em 1990, a partir do trabalho de Jakob Nielsen e Rolf Molich. A ideia era criar um método de inspeção para encontrar problemas em uma interface do usuário (SANTA ROSA \& MORAES, 2008)

A partir de uma lista de princípios reconhecidos e, preferencialmente, executada por um grupo de avaliadores - o que minimiza o risco da subjetividade excessivamente individual interferir de forma que invalide a avaliação - tem-se uma avaliação bastante completa da situação de determinada interface do ponto de vista de sua usabilidade.

Apesar de apresentada por NIELSEN \& MOLICH no início dos anos 1990, desde então diversos autores e teóricos apresentaram sua visão particular a respeito da avaliação heurística. O próprio Jakob Nielsen revisitou suas heurísticas algumas vezes, em diferentes publicações, ratificando ou retificando-as em função da ampliação dos tipos de interface passíveis de serem avaliadas e da popularização e evolução da própria internet, sua estrutura e seus recursos tecnológicos.

A avaliação heurística, constitui-se em uma técnica de inspeção de usabilidade em que especialistas, orientados por um conjunto de princípios de usabilidade conhecidos como heurística, avaliam se os elementos da interface com o usuário estão de acordo com os princípios. (PREECE, ROGERS \& SHARP, 2005, p. 430) 
Existem diversos outros autores que são referência em critérios ergonômicos para avaliação de interfaces. A seguir são apresentados, de forma resumida, alguns dos principais expoentes no assunto e suas técnicas propostas para avaliação de interface.

\subsection{Dominique Scapin e Christian Bastien}

Ainda no princípio dos anos 1990, dois pesquisadores de língua francesa, Dominique Scapin e Christian Bastien, ligados ao INRIA (Institut National de Recherche en Informatique et en Automatique) da França apresentaram uma lista de critérios para avaliação de interfaces, denominada de "critérios ergonômicos".

Esses autores mostraram que seus critérios proporcionam o aumento da sistematização dos resultados das avaliações de usabilidade de uma dada interface. Isto é, quando diferentes especialistas empregam esses critérios como ferramenta de avaliação, eles obtêm resultados mais parecidos. Eles diminuem assim, um dos inconvenientes das avaliações por especialistas, especificamente a falta de sistematização nos resultados.

São oito critérios principais, por vezes, divididos em subcritérios, conforme SANTA ROSA \& MORAES (2008):

1. Condução - Capacidade da interface em oferecer oportunidade ao usuário para a aprendizagem. Permitir que ele saiba, em tempo real, onde está naquela sequência interativa. Subdivisões: 1.1. Presteza; 1.2. Agrupamento/Distinção de Itens; 1.2.1. Agrupamento/Distinção por Localização; 1.2.2. Agrupamento/Distinção por Formato; 1.3. Feedback Imediato e 1.4. Legibilidade.

2. Carga de Trabalho - Quanto maior for a carga de trabalho ou a complexidade da tarefa, maior será a probabilidade do usuário de cometer erros. Quanto menos a atenção do usuário for desviada por informações desnecessárias, melhor ele se sairá na execução de suas tarefas. Quanto menos ações são necessárias, mais rápidas serão as interações. Subdivisões: 2.1. Brevidade; 2.1.1. Concisão; 2.1.2. Ações Mínimas e 2.2. Densidade Informacional.

3. Controle Explicito - Controle do usuário tanto sobre o processamento das ações realizadas pelo próprio usuário, quanto ao controle do usuário sobre o processamento de suas ações pelo sistema. Subdivisões: 3.1. Ações Explicitas e 3.2. Controle do Usuário.

4. Adaptabilidade - É a capacidade do sistema (ou interface) de reagir conforme o contexto e, também, as necessidades e preferências do usuário. Subdivisões: 4.1. Flexibilidade e 4.2. Experiência do Usuário.

5. Gestão de Erros - Dispositivos do sistema que visam evitar ou diminuir a ocorrência de erros. Se ocorrerem, que o sistema possibilite que o usuário facilmente os corrija. Subdivisões: 5.1. Proteção erros; 5.2. Qualidade das Mensagens de erros e 5.3. Correção erros.

6. Homogeneidade / Consistência - Forma na qual as opções na concepção da interface são mantidas idênticas em contextos idênticos e diferentes para contextos diferentes.

7. Significado de Códigos - Conformidade entre o objeto ou informação apresentados ou solicitados e sua referência. Termos pouco expressivos para o 
usuário podem ocasionar problemas de condução onde ele pode ser levado a selecionar uma opção errada.

8. Compatibilidade - Acordo que possa existir entre as características do usuário (memória, percepção, hábitos, competências, idade, expectativas, etc.) e as tarefas, de uma parte, e a organização das saídas, das entradas e do diálogo de uma dada aplicação, de outra. Coerência entre diferentes ambientes e aplicativos.

\subsection{Patrick Jordan}

Patrick W. Jordan (1998), autor e consultor britânico, com sólida formação em psicologia, que escreve sobre estratégia em design, marketing e branding é, também, uma referencia na avaliação de interfaces e apresenta seus dez princípios para design com usabilidade:

1. Coerência - Respeitar a convenção de que características similares devem ser realizadas da mesma forma. Incoerências levam o usuário a cometer erros.

2. Compatibilidade - Projetar para que o produto corresponda à expectativa do usuário, sem deixar de lado o conhecimento que o usuário tem do mundo real.

3. Consideração sobre a habilidade do usuário - Levar em conta a habilidade e a experiência pregressa do usuário.

4. Retorno das ações / feedback - A interface deve oferecer reações claras com relação às ações que o usuário realiza.

5. Prevenção de erro e recuperação - A interface pode ser projetada para minimizar a ocorrência de erros e o usuário recuperar, de forma rápida e fácil, qualquer erro que cometa.

6. Controle do usuário - O usuário dever ter o máximo de controle o possível sobre as interações que realiza com o sistema.

7. Clareza visual - A informação (incluindo rótulos e feedback) deve ser acessada fácil e rapidamente, sem obstáculos.

8. Priorização da funcionalidade e da informação - Se o produto ou sistema fornece muitas informações, deve-se priorizar algumas características ao projetar a interface, conforme a frequência de uso ou a importância da função.

9. Transferência adequada de tecnologia - $O$ uso de tecnologias que foram desenvolvidas para um fim e que acabam sendo aplicadas para outro podem ser úteis e benéficas. Mas se utilizadas sem o devido estudo e cuidado, podem gerar problemas ao produto ou sistema.

10. Explicitação - Quanto mais explicita e simples a forma de operar um produto, melhor.

\subsection{Jakob Nielsen}

Como demonstrado anteriormente, dentre os autores que trabalham a avaliação heurística pode-se ressaltar o trabalho de Jakob Nielsen, que por mais de duas décadas tem pesquisado e divulgado seu trabalho como especialista em usabilidade, especialmente usabilidade na web. Como precursor da avaliação 
heurística, Nielsen não apenas cunhou este termo, ainda nos anos 1990, mas também tornou a principal referência no assunto.

Em seu artigo How to conduct a Heuristic Evaluation (1995), Nielsen afirma que a análise heurística é um método rápido e barato para avaliar a usabilidade de um sistema. Segundo o autor, recomenda-se que a análise seja realizada por especialistas em usabilidade, porém nada impede que usuários estudem os critérios de avaliação e realizem a análise.

Com relação à quantidade de avaliadores, o autor afirma que a tarefa de avaliar uma interface por meio de heurísticas pode ser realizada de forma individual, mas a experiência indica que os resultados são mais pobres neste caso.

Estudos indicaram que avaliadores individuais encontram, em média, apenas $35 \%$ dos problemas de usabilidade em suas análises. Isto porque diferentes avaliadores tendem a encontrar problemas diferentes - há uma substancial melhora da performance quando se utiliza vários avaliadores (NIELSEN, 1995).

As heurísticas recomendadas por NIELSEN \& MOLICH (1990), segundo o próprio NIELSEN (1995b) foram desenvolvidas para avaliação heurística, em colaboração com Rolf Molich em 1990. Desde então, elas foram refinadas com base em uma análise fatorial de 249 problemas de usabilidade para derivar um conjunto de heurísticas com o máximo poder explicativo, resultando num conjunto revisto de heurísticas.

Assim, devido a diferença entre a lista das heurísticas apresentadas por autores como SANTA ROSA \& MORAES (2008) e SALMON (2004), apresentam-se aqui as heurísticas descritas pelo próprio NIELSEN (1995b), em seu artigo "10 Usability Heuristics for User Interface Design". São elas:

1. Visibilidade do status do sistema - O sistema deve, sempre, manter o usuário informado sobre o que está acontecendo, fornecendo um feedback apropriado, num tempo razoável.

2. Equivalência entre o sistema e o mundo real - $O$ sistema deve falar a linguagem do usuário, com palavras, frases e conceitos familiares a ele, ao invés de utilizar termos técnicos orientados ao sistema. Seguir convenções do mundo real, fazendo com que a informação seja exibida em sua ordem lógica e natural.

3. Controle do usuário e liberdade - Usuários frequentemente escolhem erroneamente funções do sistema, e necessitarão uma clara e demarcada "saída de emergência" para sair de um estado indesejado sem a necessidade de passar por um caminho extenso e complexo. O sistema deve oferecer suporte para ações como: desfazer e refazer.

4. Consistência e padrões - Usuários não tem que adivinhar quando palavras, situações ou ações diferentes significam a mesma coisa. Siga convenções.

5. Prevenção de erros - Melhor do que boas mensagens de ero, é o cuidado para prevenir um problema, antes que ele aconteça, em primeiro lugar. Solicitar a confirmação do usuário antes de executar comandos ajuda a evitar diversas escolhas feitas de forma equivocada.

6. Reconhecimento ao invés de memorização - Minimizar a carga de memória do usuário, tornando objetos, ações e opções visíveis. O usuário não deve ter que lembrar da informação de uma parte do diálogo para outra. As instruções para 
usar o sistema devem ser visíveis e facilmente localizáveis, sempre que apropriado.

7. Flexibilidade e eficiência de uso - Aceleradores de tarefa, invisíveis aos usuários novatos podem oferecer mais rapidez na tarefa para usuários experientes, assim como o sistema deve atender tanto usuários inexperientes, quanto experientes.

8. Estética e design minimalista - Diálogos não devem conter informação que não seja relevante. Cada informação irrelevante compete com as relevantes e reduzem sua visibilidade.

9. Ajudar os usuários a reconhecer, diagnosticar e recuperar ações erradas Mensagens de erro devem ser expressas em uma linguagem simples (sem códigos), indicando precisamente o problema, e sugerindo, construtivamente, uma solução.

10. Ajuda e documentação - Mesmo tendo como meta que o sistema deve poder ser utilizado sem documentação, pode ser necessário fornecer ajuda e documentação. Qualquer informação neste sentido deve ser fácil de buscar, focada na tarefa do usuário, listando passos concretos para ser levada adiante e sem ser grande demais.

\section{COMPARATIVO ENTRE AS AVALIAÇÕES HEURÍSTICAS}

A seguir, é apresentado um quadro comparativo, a título de ilustração, entre as heurísticas (ou critérios ergonômicos) descritas pelos principais autores da área, onde estas heurísticas são agrupadas por similaridade, tomando como referência as heurísticas de NIELSEN \& MOLICH (1990); NIELSEN (1995b).

Quadro 1 - Comparativo das heurísticas (critérios ergonômicos), a partir de NIELSEN \& MOLICH (1990), NIELSEN (1995b); SCAPIN \& BASTIEN (1993) e JORDAN (1998).

\begin{tabular}{|c|c|c|}
\hline NIELSEN \& MOLICH & SCAPIN \& BASTIEN & JORDAN \\
\hline $\begin{array}{l}\text { 1. Visibilidade do status do } \\
\text { sistema }\end{array}$ & $\begin{array}{l}\text { 1. Condução } \\
\text { 3. Controle explícito }\end{array}$ & $\begin{array}{l}\text { 4. Retorno das ações / } \\
\text { feedback } \\
\text { 7. Clareza visual }\end{array}$ \\
\hline $\begin{array}{l}\text { 2. Equivalência entre o } \\
\text { sistema e o mundo real }\end{array}$ & $\begin{array}{l}\text { 6. Homogeneidade / } \\
\text { consistência } \\
\text { 8. Compatibilidade }\end{array}$ & 2. Compatibilidade \\
\hline $\begin{array}{l}\text { 3. Controle do usuário e } \\
\text { liberdade }\end{array}$ & $\begin{array}{l}\text { 1. Condução } \\
\text { 3. Controle explícito }\end{array}$ & 6. Controle do usuário \\
\hline 4. Consistência e padrões & $\begin{array}{l}\text { 6. Homogeneidade / } \\
\text { consistência }\end{array}$ & $\begin{array}{l}\text { 1. Coerência } \\
\text { 9. Transferência adequada de } \\
\text { tecnologia }\end{array}$ \\
\hline 5. Prevenção de erros & 5. Gestão de erros & $\begin{array}{l}\text { 1. Coerência } \\
\text { 5. Prevenção de erro e } \\
\text { recuperação }\end{array}$ \\
\hline $\begin{array}{l}\text { 6. Reconhecimento ao invés } \\
\text { de memorização }\end{array}$ & $\begin{array}{l}\text { 2. Carga de trabalho } \\
\text { 7. Significados de códigos }\end{array}$ & $\begin{array}{l}\text { 3. Consideração sobre a } \\
\text { habilidade do usuário } \\
\text { 10. Explicitação }\end{array}$ \\
\hline $\begin{array}{l}\text { 7. Flexibilidade e eficiência de } \\
\text { uso }\end{array}$ & 4. Adaptabilidade & $\begin{array}{l}\text { 3. Consideração sobre a } \\
\text { habilidade do usuário }\end{array}$ \\
\hline
\end{tabular}




\begin{tabular}{|l|l|l|}
\hline & & $\begin{array}{l}\text { 8. Priorização da } \\
\text { funcionalidade e da } \\
\text { informação }\end{array}$ \\
\hline $\begin{array}{l}\text { 8. Estética e design } \\
\text { minimalista }\end{array}$ & $\begin{array}{l}\text { 1. Condução } \\
\text { 2. Carga de trabalho }\end{array}$ & 7. Clareza visual \\
\hline $\begin{array}{l}\text { 9. Ajudar os usuários a } \\
\text { reconhecer, diagnosticar e } \\
\text { recuperar ações erradas }\end{array}$ & 5. Gestão de erros & $\begin{array}{l}\text { 5. Prevenção de erro e } \\
\text { recuperação }\end{array}$ \\
\hline 10. Ajuda e documentação & 4. Adaptabilidade & 10. Explicitação \\
\hline
\end{tabular}

Tal quadro pode e será aprofundado, em pesquisas posteriores, no que tange à relação entre os autores. No entanto, mesmo nesta breve comparação, é possível perceber - ao listar algumas das heurísticas (ou critérios ergonômicos) mais utilizadas na avaliação de interfaces - que são elencados itens bastante similares, apresentando preocupações semelhantes no tocante à interação entre o usuário e a interface.

Qualquer uma das técnicas apresentadas, ainda que resumidamente, neste artigo seriam uma ferramenta bastante adequada ao que se propõe nesse trabalho. Assim, utilizar-se-ão as heurísticas de Jakob Nielsen, como referência - e por convenção - para compor a proposta deste artigo. No entanto, não há, de forma alguma, impedimento para que se utilize qualquer outra referência, em termos de técnica e/ou autor para se realizar a avaliação proposta.

\section{COMO AS HEURÍSTICAS PODEM CONTRIBUIR COM OS MÉTODOS BASEADOS EM GARRETT}

Na tabela a seguir, uma proposta de relacionamento entre as recomendações de GARRETT (2003) para o plano Superfície e onde as heurísticas de NIELSEN \& MOLICH (1990); NIELSEN (1995b) podem ser inseridas como avaliação da interface ainda na fase projetual - conforme sugere SALMON (2004):

Quadro 2 - Resumo da aplicação das heurísticas ao método de Garrett, a partir de NIELSEN \& MOLICH (1990), NIELSEN (1995b) e GARRETT (2003).

\begin{tabular}{|l|l|}
\hline $\begin{array}{l}\text { Recomendações de } \\
\text { GARRETT (2003) } \\
\text { para o plano Superfície }\end{array}$ & $\begin{array}{l}\text { Heurísticas de NIELSEN \& MOLICH (1990); NIELSEN (1995b) que } \\
\text { podem ser aplicadas para verificação e/ou aprimoramento }\end{array}$ \\
\hline $\begin{array}{l}\text { Follow the eye } \\
\text { (Siga o olho) }\end{array}$ & $\begin{array}{l}\text { 3. Controle do usuário e liberdade } \\
\text { 5. Prevenção erros }\end{array}$ \\
\hline $\begin{array}{l}\text { Contrast and uniformity } \\
\text { (Contraste e } \\
\text { uniformidade) }\end{array}$ & $\begin{array}{l}\text { 2. Equivalência entre o sistema e o mundo real } \\
\text { 4. Consistência e padrões } \\
\text { 8. Estética e design minimalista }\end{array}$ \\
\hline $\begin{array}{l}\text { Internal and external } \\
\text { consistency } \\
\text { (Coerência interna e } \\
\text { externa) }\end{array}$ & $\begin{array}{l}\text { 1. Visibilidade do status do sistema } \\
\text { 2. Equivalência entre o sistema e o mundo real } \\
\text { 4. Consistência e padrões } \\
\text { 6. Reconhecimento ao invés de memorização } \\
\text { 7. Flexibilidade e eficiência de uso } \\
\text { 9. Ajudar os usuários a reconhecer, diagnosticar e recuperar ações } \\
\text { erradas }\end{array}$ \\
\hline Color Palettes and & 4. Consistência e padrões \\
\hline
\end{tabular}




\begin{tabular}{|c|c|}
\hline $\begin{array}{l}\text { Typography } \\
\text { (Paletas de cores e } \\
\text { tipografia) }\end{array}$ & 8. Estética e design minimalista \\
\hline $\begin{array}{l}\text { Design Comps and Style } \\
\text { Guides } \\
\text { (Protótipos e guia de } \\
\text { estilo) }\end{array}$ & $\begin{array}{l}\text { 2. Equivalência entre o sistema e o mundo real } \\
\text { 4. Consistência e padrões } \\
\text { 8. Estética e design minimalista } \\
\text { 10. Ajuda e documentação }\end{array}$ \\
\hline
\end{tabular}

Além de incluir um plano de execução no método original, MEURER \& SZABLUK (2009) também redesenharam alguns processos da metodologia original. Em relação à etapa de Superfície, por exemplo, os autores a rebatizaram de 'Estética' e, de fato, pautaram suas recomendações em questões de cunho essencialmente estético-formal, deixando de lado abordagens ligadas à avaliação da interface propriamente dita:

\section{Quadro 3 - Resumo da etapa Estética, a partir de MEURER \& SZABLUK (2009)}

\begin{tabular}{|l|l|}
\hline $\begin{array}{l}\text { Estudo e definição } \\
\text { das malhas }\end{array}$ & $\begin{array}{l}\text { O Projeto E indica o uso de malhas diagramacionais para elaboração do } \\
\text { leiaute das telas. Elas são oriundas das mais diferentes teorias } \\
\text { filosóficas e geométricas, e podem até mesmo ser geradas por } \\
\text { aplicativos na world wide web. }\end{array}$ \\
\hline $\begin{array}{l}\text { Composição e } \\
\text { diagramação }\end{array}$ & $\begin{array}{l}\text { Devem ser feitas sempre em concordância com as exigências e } \\
\text { restrições físicas às quais a IGA (interface gráfica amigável) será } \\
\text { submetida. No caso da world wide web, variações de configuração e } \\
\text { resolução de tela devem ser consideradas sempre, para que o layout } \\
\text { possa se adaptar facilmente a grandes diferenças de configuração }\end{array}$ \\
\hline $\begin{array}{l}\text { Identidade gráfico- } \\
\text { visual }\end{array}$ & $\begin{array}{l}\text { Durante a composição do layout das telas de um produto dígito-virtual, } \\
\text { os elementos da identidade gráfico-visual devem ser criteriosamente } \\
\text { desenhados e introduzidos na interface. Os elementos que definem o } \\
\text { padrão visual da IGA são: (a) Logografia; (b) Cromografia; (c) Tipografia; } \\
\text { (d) Pictografia e (e) Iconografia. }\end{array}$ \\
\hline
\end{tabular}

Assim, para o método proposto por MEURER \& SZABLUK, a Avaliação Heurística pode ser aplicada nesta etapa integralmente, com o intuito tornar o resultado da etapa mais confiável, uma vez que está estará baseada em uma técnica de avaliação consolidada e não somente nas impressões subjetivas do grupo de desenvolvedores daquele projeto.

\section{CONCLUSÃO}

Embora bastante difundido e utilizado, o método de projeto centrado no usuário de J. J. Garrett pouco refere-se à uma avaliação sistematizada, em especial em sua etapa "plano de Superfície". Ainda que este não seja o objetivo e nem o escopo proposto pela metodologia em questão, faz-se necessário refletir a respeito do ganho que se pode ter ao aliar o referido método de projeto com uma técnica de avaliação de interface consolidada.

A ideia de ganho surge ao propor a aplicação de técnica de avaliação de interface para a etapa de Superfície, em GARRETT (2003) - ou Estética, em MEURER \& SZABLUK (2009) - em função da provável redução do retrabalho e, por consequência, 
do custo de projeto, de uma maneira geral. Adotando uma técnica consolidada de avaliação, conforme exposto anteriormente, obtém-se uma garantia de muitos desses problemas que seriam percebidos apenas mais adiante, quando o sistema estivesse sendo utilizado pelos usuários finais do produto, poderiam ser antecipados ainda na fase de projeto.

Para tanto, sugere-se que a técnica utilizada seja a chamada Avaliação Heurística proposta por NIELSEN \& MOLICH (1990). Entre as vantagens do uso desta técnica, estão aquelas citadas por SALMON (2004), sejam elas: a simplicidade e a velocidade de aplicação, resultados relevantes e de uso imediato, uso de recursos extremamente baixo e a possibilidade de uso durante todo o processo projetivo. É provável que, se movermos a avaliação da interface para dentro do processo projetivo, possamos antecipar problemas de usabilidade que não tenham sido detectados pelos projetistas, muito em função de sua proximidade do objeto projetado, inclusive.

Desta forma, antecipar a detecção de erros e problemas na interface, além de uma provável economia de recursos de desenvolvimento como sugerido anteriormente, pode, ainda, evitar que muitos problemas de projeto, que deterioram a usabilidade de determinada interface, cheguem ao produto acabado, acessível ao usuário final. Menos problemas na interação com o usuário final representa menos frustração de uso. Menos frustração no uso representa menos constrangimento ergonômico. Menos constrangimentos significa um usuário realizando sua tarefa de forma mais eficiente.

\section{REFERÊNCIAS}

BASTIEN, C.; SCAPIN, D.. Ergonomic Criteria for the Evaluation of Human Computer Interfaces. INRIA, 1993.

BOMFIM, G. A.. Metodologia para o desenvolvimento de projetos. João Pessoa: Ed. Universitária/UFPB, 1995.

GARRETT, J.J.. The Elements of User Experience. New York, NY - USA: AIGA American Institute of Graphic Arts, 2003.

The Elements of User Experience: User-Centered Design for the Web and Beyond. 2nd Ed. Berkeley: New Riders. 2011.

JORDAN, P. W. An Introduction to Usability. Londres: Taylor \& Francis, 1998.

KALBACH, J.. Design de Navegação Web: Otimizando a experiência do usuário. Porto Alegre: Bookman, 2009.

LABIUTIL. Laboratório de Utilizabilidade da Informática / Universidade Federal de Santa Catarina. Disponível em: <http://www.labiutil.inf.ufsc.br/>. Acesso em: 14 nov. 2013.

LYNCH, P.J.; HORTON, S.. Web Style Guide: basic design principles for creating web sites. Kendallville: Yale University, 2008.

MEURER, H.; SZABLUK, D.. Projeto E: aspectos metodológicos para o desenvolvimento de projetos dígito-virtuais. In: 9 ERGODESIGN | 9 USIHC, 2009, Curitiba. Anais do 9 ERGODESIGN | 9 USIHC, 2009.

Projeto E: aspectos metodológicos para o desenvolvimento de projetos dígito-virtuais. Ação Ergonômica, v. 5, p. 1-10, 2010.

NIELSEN, J.; MOLICH, R. Heuristic evaluation of user interfaces. In J. C Chew \& J. Whiteside (eds). Empowering people: CHI 90 Conference Proceedings (pp. 249 - 256) Monterey, CA: ACM Press, 1990. 
NIELSEN, J. How to conduct a Heuristic Evaluation (Jan, 1995). Disponível em: <http://www.nngroup.com/articles/how-to-conduct-a-heuristic-evaluation/>. Acesso em 6 out 2013.

NIELSEN, J. 10 Usability Heuristics for User Interface Design (Jan, 1995). Disponível em <http://www.nngroup.com/articles/ten-usability-heuristics/>. Acesso em 14 nov 2013. PREECE, J.; ROGERS, Y.; SHARP, H.. Design de interação: além da interação homemcomputador, Porto Alegre, RS: Bookman, 2005.

SALMON, P. Human Factors Design and Evaluation Methods Review. Southampton: $\mathrm{HFi}$ - DTC, 2004.

SANTA ROSA, J.G.; MORAES, A. Avaliação e projeto no design de interfaces. Teresópolis, RJ: 2AB, 2008. 